Ann. Sci. forest., I976, 33 (3), I6I-I75.

\title{
ÉTUDE EXPÉRIMENTALE DE LA CONTAMINATION POLLINIQUE DU VERGER A GRAINE DE PIN MARITIME DE SORE (LANDES)
}

\author{
J. Ph. Castaing et Pii. Vergeron \\ Association Forêt-Cellulose, \\ 63, Rue E. Renan, \\ 33000 Bordeaux \\ Laboratoire d'Amélioration des Conifères, \\ Centre de Recherches de Bordeaux, I. N. R. A., \\ Pierroton Cestas, \\ 33610 Gazinet
}

\section{RÉSUMÉ}

L'installation d'un verger à graine de familles de pin maritime à Sore, nécessite une étude approfondie de la dispersion du pollen de cette espèce dans le massif landais.

Par piégeage du pollen, soit sur toute la surface du verger, soit le long de certains axes traversant ce dernier, les auteurs ont constaté que les peuplements directement voisins sont impliqués dans la majeure partic du phénomène de contamination du verger.

Il est possible de construire à l'aide de la formule de Rombakis une représentation mathématique simple de la ligne de vol moyenne d'un nuage de pollen. La distance maximum probable de dispersion se situe pour le pin maritime, entre 500 et $\mathbf{I}$ ooo mètres. Il est ainsi possible de proposer une gestion particulière des alentours d'un verger à graine.

\section{I. - INTRODUC'TION}

Les programmes récents d'amélioration génétique du Pin maritime de la forêt landaise ont abouti à l'installation, dans ce massif forestier, de vergers à graine de familles. Les tests de descendances, permettant le principal travail de sélection sont, en effet, implantés au sein du massif utilisateur. L'existence possible d'interaction génotype-milieu rend obligatoire la mise en place du verger à graine à l'intérieur de la région landaise. L'étude approfondie de la dispersion du pollen de pin maritime est ainsi devenue indispensable. 
La contamination d'un verger à graine par du " pollen sauvage » entraîne inévitablement une baisse de gain génétique pour chaque caractère en cours d'amélioration. BARADAT (I970) a estimé la diminution de gain génétique en fonction du taux de pollution pollinique et de la valeur génétique des peuplements émettant le pollen exogène. Il serait donc intéressant de connaître avec suffisamment de précision 1'origine du pollen étranger pouvant se déposer sur les inflorescences femelles des arbres du verger et la proportion suivant laquelle il entre en compétition avec le pollen endogène du verger.

Ce travail porte uniquement sur le premier point.

A Sore (Landes), un verger à graine de semis est en cours d'installation. Un terrain de r2o hectares a été reten11. Sa forme, grossièrement circulaire, permet de réduire au maximum son périmètre. Aussi, les échanges de pollen avec le voisinage s'en trouvent théoriquement limités (BARADAT et al., I970).

Les tranches annuelles d'installation sont disposées de façon concentrique, de telle sorte que les plus anciennes occupent la périphérie, le centre étant réservé à la dernière. Ainsi, les tranches internes qui seront constituées de familles issues de parents hautement sélectionnés devraient être protégées par les tranches plus anciennes qui forment un écran vis-à-vis du pollen exogène.

Les peuplements adultes proches du verger se trouvent au Nord et à 1'Est de ce dernier. A l'Ouest, ils sont beaucoup plus jeunes (9 ans en rg69).

En I975, la moitié du verger à graine est installé. Les plants les plus anciens ont huit ans et émettent du pollen depuis 2 ans (première émission en I973).

Cependant, jusqu'en I972, nos études se sont déroulées alors que les premiers plants du verger étaient encore de petite taille et ne portaient pas d'inflorescences. La contamination par les peuplements extérieurs a donc été étudiée de façon isolée, la surface du verger à graine étant considérée comme libre de toute source endogène.

Sur le plan dynamique, les facteurs intervenant dans les phénomènes de transport du pollen sont nombreux, complexes et très souvent variables dans l'espace et dans le temps. Cette instabilité des facteurs de dispersion, l'émission simultanée de nuages polliniques d'origines différentes et l'application difficile des méthodes statistiques aux résultats sont autant de faits rendant les généralisations souvent délicates. Seuls des recoupements de résultats d'une région à l'autre et surtout d'une année à l'autre ont le plus souvent permis d'arriver à nos conclusions. Ces dernières sont d'ailleurs confirmées par des résultats d'expériences obtenues à l'extérieur du verger de Sore.

\section{2. - MATÉRIEI E'T MÉTHODES}

2. I. - Méthodes de Piégeage

Les pièges utilisés sont constitués de plaquettes d'aluminium de $85 \mathrm{~mm}$ de long sur 25 de

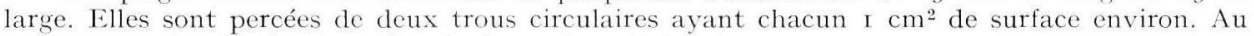
moment de l'utilisation, une bande de papier adhésif transparent est collée sur le dos de cette piaquette. La face exposée est donc adhésive au niveau des perforations (Illy et Sopena, I963).

Ces pièges sont fixés à la partie supéricure de piquets en bois, de section carrée et de I,5o de hauteur. Ces piquets sont orientés au moment de leur installation, une face vers le Nord: quatre pièges sont utilisés par piquet, chacun faisant face à un des points cardinaux.

La période de dissémination sur l'ensemble du massif landais dure cnviron une trentaine de 
jours. Au niveau d'une région donnée du massif, la durée de la floraison n'excède guère une semaine (ILLy et Sopena 1963). Les séries de pièges ont été renouvelées tous les quatre jours, jusqu'en I97I, tous les trois jours à partir de 1972. Il a été vérifié que le film utilisé reste adhésit pendant 5 à 6 jours.

Ces types de pièges ont des inconvénients théoriques certains. Gregory, I945 ; SARVAS, I962, ont montré que l'efficacité optimale était obtenue avec des pièges de petite dimension et de forme sphérique. Ils permettent cependant des comptages rapides, favorisant l'installation d'expériences importantes (CAStaing, Vergerox, I973).

\section{2. - Méthodes de comptage}

Après exposition, les pièges ont été observés d'abord à la loupe binoculaire (1969), ensuite sur l'écran d'une microvisionneuse. Dans le premier cas, les grains de pollen ont été comptés sur un certain nombre de champs microscopiques dont la surface totale constituait un échantillonnage clu dixième de la surface exposée. A partir de I970, au moment du comptage, chaque piège est recouvert d'un cache en carton ajouré. Huit perforations rectangulaires sont ainsi régulièrement disposées sur la surface de piégeage. Chaque perforation correspond à deux champs optiques de la visionneuse et les seize champs observés représentent environ le quart de la surface adhésive utile. Pour chaque piège on additionne les nombres de grains de pollen comptés au niveau de chacune des perforations.

Les méthodes de comptage ont donc évolué au cours des années d'étude successives de telle sorte qu'il est impossible de comparer les résultats absolus des comptages d’une saison de dispersion à une autre. Par contre, on peut très légitimement comparer les résultats relatifs, ce qui est l'essentiel.

\section{3. - Dispositifs d'expérience}

Ils ont également évolué dans le temps; nous pouvons distinguer trois périodes. 2. 3. I. Études préliminaires $(1969,1970$ et 19\%1).

Cinq piquets dispersés sur toute la surface du verger numérotés de I à 5 (fig. I).

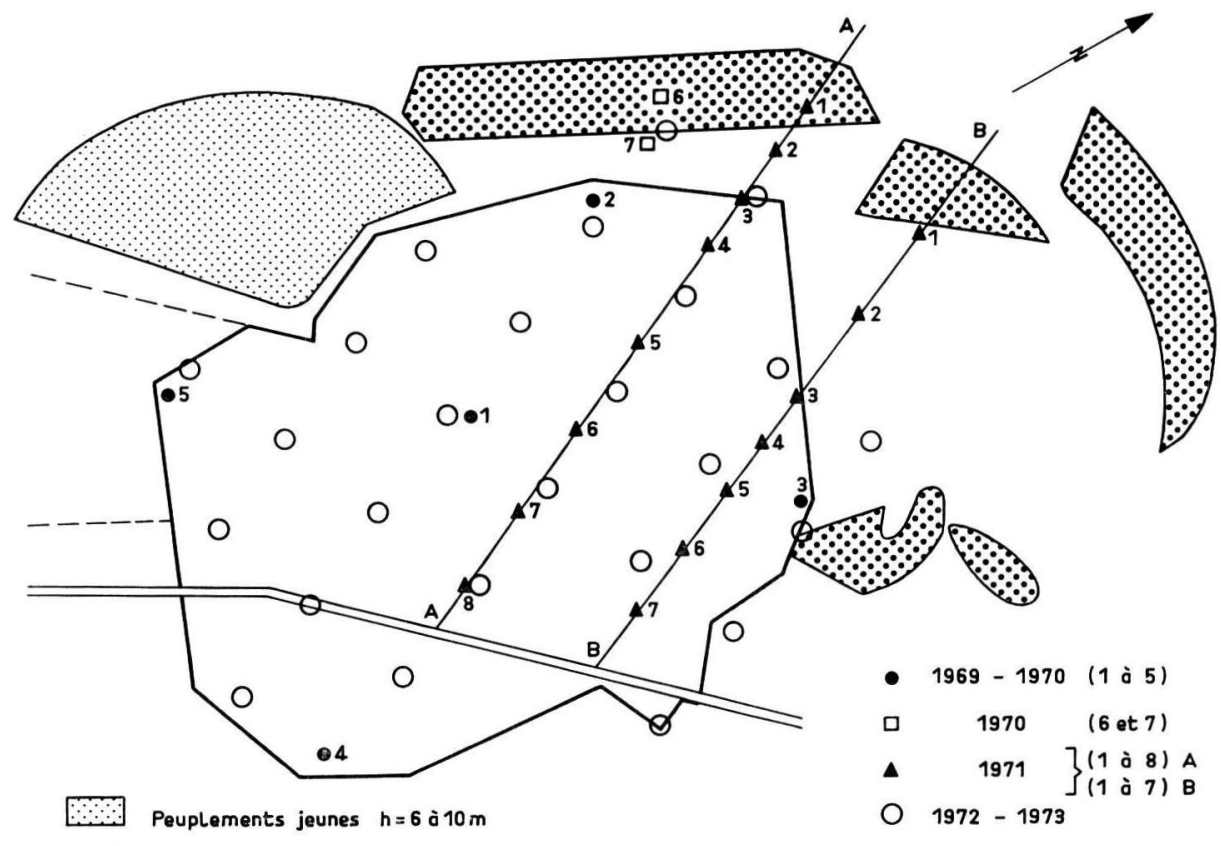

$\because \because \because$ Peuplements adultes $h=20$ ò $25 \mathrm{~m}$

Echelle : $1 / 20000$

FIG. I. - Plan du verger à graines de Sore (Landes). Points de piégeages de 19619 à 197.3

Fig. I. - Map of seed orchard of Sore (Landes. Trapping sites from 1969 to 197.3 
A partir de 1970 , deux piquets $n^{\circ} 6$ et 7 ont été ajoutés en dehors du verger, au Nord, le premier à l'intérieur d'un massif adulte voisin, le second à la lisière de ce même massif du côté verger (fig. I).

\section{3.2. - Etude de transect (1970 et 1971).}

Les piquets de I à 5 sont maintenus. Nous avons ajouté en I97 I deux lignes de piquets NordEst à Sud Sud-Ouest (fig. I).

- Ligne A : presque diagonale du verger : 8 points de A I à . Is, A I étant à l'extérieur du verger dans le massif adulte voisin; les intervalles entre $A_{\text {I }}$ et $A+$ sont égaux à roo mètres, entre $\mathrm{A}_{4}$ et $\mathrm{A} 8$ à $200 \mathrm{~m}$.

- Ligne B : traverse la partie Est du verger; 7 points de $B$ I à B 7 . Les intervalles de B I à B 5 sont égaux à Ioo mètres, ceux de $B 5$ à $B 7$ à 200 mètres.

\section{3.3. - Essai de cartographie de la contamination du verger à graine par le pollen étranger} (1972 ct 1973).

Vingt-cinq piquets ont été régulièrement disposés sur toute la surface du verger a fin d'établir une véritable cartographie de la contamination pollinique, surtout au moment de la floraison du voisinage immédiat (fig. I).

\section{3. - PRINCIPES GÉNÉRAUX D'ÉTUDE \\ DE LA DISPERSION DE PAR'TICULES DANS L'A'TMOSPHÈRE}

Jusqu'à ces dernières années, ce sont les représentations théoriques de SutTon (I947) reprises par STRAND (I957) qui ont été utilisées par les chercheurs travaillant sur la dispersion des grains de pollen et des spores dans l'atmosphère. De l'aveu même de STRAND cette application reste difficile et les écarts entre les courbes théoriques de vol et les résultats de piégeages sont très importants. WRIGHT (I953) après STEPANOV (I935) caractérise une dispersion en calculant un écart type classique. Or, les chiffres classiquement observés, même en dispersion expérimentale (lacher artificiel de spores) ne sont jamais distribués normalement.

Le modèle de Rombakis ( I947) a été utilisé par SchrodTen en I960. Il étudie une ligne probable de vol, telle que si nous considérons un point $\mathrm{P}$ de la courbe, un grain de pollen quelconque a autant de chance de se trouver en dessus qu'en dessous de cette courbe.

L'équation de la courbe est la suivante :

$$
z=0.4769 \sqrt{\frac{4 \bar{A} x}{\mathrm{~V} \delta}-\frac{c x}{v}}
$$

C'est une équation simple dans laquelle:

$z=$ altitude à une distance $x$ de la source en mètre ;

$x=$ distance parcourue depuis la source en mètre ;

$A=$ coefficient d'échange atmosphérique en $\mathrm{g} \cdot \mathrm{m} / \mathrm{cms}$.

C'est la quantité de matière échangeable à travers une surface donnée à une altitude $\mathrm{Z}$ pendant l'unité de temps :

$\delta=$ masse spécifique de l'air

$\mathrm{V}=$ vitesse du vent en $\mathrm{m} / \mathrm{S}$ :

$\mathrm{C}=$ vitesse limite de chute dans 1'air calme des particules étudiées en $\mathrm{m} / \mathrm{s}$. 
Les facteurs fixes sont :

$\delta$ : masse spécifique de l'air ;

C : la vitesse limite de chute de la particule. Elle a été mesurée expérimentalement par les auteurs, suivant la méthode de Durham (I946). Ëlle est égale à $0,17 \mathrm{~m} / \mathrm{s}$ pour le pin maritime (Castaing et Vergeron, I973).

Les facteurs variables sont :

$\mathrm{V}$ : vitesse du vent ;

A : coefficient d'échange atmosphérique.

Selon les météorologistes, il varie en fonction de 1'altitude.

Aux meilleures approximations il est égal à :

$$
\begin{aligned}
& \text { I } g \cdot \mathrm{m} / \mathrm{cm} \cdot \mathrm{s} \text { de } 0 \text { à Io mètres d'altitude } \\
& \text { Io } g \cdot \mathrm{m} / \mathrm{cm} \cdot \mathrm{s} \text { de ro à IoO mètres d'altitude } \\
& 50 \mathrm{~g} \cdot \mathrm{m} / \mathrm{cm} \cdot \mathrm{s} \text { de Ioo à } 500 \text { mètres d'altitude }
\end{aligned}
$$

Les phénomènes que nous étudions ayant leur source au niveau de la cime des pins adultes, nous pourrons donc fixer le coefficient d'échange à $\mathrm{I} O \mathrm{~g} \cdot \mathrm{m} / \mathrm{cm} \cdot \mathrm{s}$.

Énfin, nous pouvons tirer de l'équation (I) la distance moyenne probable parcourue

Lorsque cette distance $\mathrm{X}$ est atteinte :

$$
z=0
$$

d'où :

$$
\mathrm{X}=0,9 \mathrm{I} \begin{aligned}
& \mathrm{AV} \\
& \mathrm{C}^{2} \delta
\end{aligned}
$$

Cette distance moyenne probable est donc :

- proportionnelle au coefficient d'échange atmosphérique et à la vitesse du vent ;

- inversement proportionnelle au carré de la vitesse limite de chute en air calme de la particule. Autrement dit, la distance moyenne probable parcourue, est d'autant plus longue que le vent est plus fort et la turbulence élevée et que la vitesse limite de chute est faible. Ceci semble cohérent et il sera intéressant de voir s'il nous est possible d'appliquer le modèle théorique de Rombakis aux résultats expérimentaux obtenus sur le verger à graine de pin maritime de Sore.

\section{t. - RÉSULTATS}

I1 est à remarquer que nous n’étudions alors que le parcours probable du mode et non de l'ensemble de la population constituée par les grains de pollen du nuage émis.

\section{†. I. Études préliminaires: années 1969, 1970 et 1971}

\section{I.I. Phénomène général.}

Nous pouvons exprimer pour chaque période de ces trois années la quantité de pollen reçue en pourcentage de la quantité totale de pollen de l'année. 


\begin{tabular}{c|c|c|c|c|c|c|c|c|c|c|c|c}
\hline \hline Dates & $24-4$ & $2,-5-4$ & $28-4$ & $29-4$ & $2-5$ & $3-5$ & $6-5$ & $7-5$ & $10-5$ & $11-5$ & $16-5$ & $20-5$ \\
\hline 1969 & $-3,2$ & & 42,4 & & 49,3 & & 4,7 & & 0,4 & & & \\
\hline 1970 & & 10,5 & & 5,4 & & 11,0 & & 49,6 & & 20,7 & 2,8 & \\
\hline 1971 & & 0,1 & & 1,8 & & 19,2 & & 30,7 & & 36,8 & 8,7 & 2,7 \\
\hline
\end{tabular}

La date de dissémination locale a varié selon l'année. 'Très précoce et brutale en I969, (du 24-4 au 2-5) cumulant 92 p. Ioo du pollen total reçu pendant toute la campagne, plus tardive en I970 mais plus étalée et plus irrégulière en I97 I à cause des conditions atmosphériques (temps très pluvieux).

Au moment de la floraison locale, les vents avaient pour tendance générale.

- Ouest en rg69;

- Nord-Ouest en I970;

- Nord-Est en I97I.

4. I.2. - Étude de l'origine du pollen capté sur le verger à graine.

Nous donnerons pour les trois années successives les pourcentages de pollen capté selon les 4 directions cardinales. Nous obtenons :

\begin{tabular}{l|c|c|c|c}
\hline \hline & $\mathrm{N}$ & $\mathrm{S}$ & $\mathrm{I}$ & $\mathrm{W}$ \\
& & & & \\
& & & & \\
$1969 \ldots \ldots \ldots \ldots$ & 35,0 & 15,2 & 39,1 & 10,7 \\
$1970 \ldots \ldots \ldots \ldots$ & $2.2,0$ & 10,5 & 18,6 & 18,9 \\
$1971 \ldots \ldots \ldots \ldots$ & 19,8 & 13,1 & 34,1 & 33,0 \\
\hline
\end{tabular}

C'est l'apport cumulé du Nord et de 1'Est qui est le plus important au cours des trois années. Celui de l'Ouest semble en progression régulière. Eit cela indépendamment de la direction des vents dominants.

Ce calcul de pourcentage est effectué sur le nombre total de grains de pollen piégés au cours de toute la période de dissémination landaise. Ce qui en fait nous intéresse plus particulièrement ce sont les mêmes données caculées au moment de la floraison locale, c'est-à-dire au moment de la réceptivité des fleurs femelles.

Nous obtenons alors :

\begin{tabular}{|c|c|c|c|c|}
\hline & N & S & $\mathrm{I}:$ & II \\
\hline 1969. & 36,6 & 21,5 & 33,4 & 8,5 \\
\hline $1970 \ldots \ldots \ldots$ & 13,6 & 9,3 & $6 i^{\prime}, \overline{5}$ & $1 \geq 6$ \\
\hline $1971 \ldots \ldots \ldots$ & 9,5 & $1: 3,7$ & $5 ., 3$ & 21,5 \\
\hline
\end{tabular}


Le pollen venu de 1'Esst reste très dominant, la proportion venue de l'Ouest montre toujours la même progression que celle obtenue plus haut.

$$
\text { 4. 2. - Étude des transects (19\%0 et } 19 \% 1)
$$

4. 2.I. - Étude au cours de l'année $19 \% 0$.

I,es points $6,7,2$, I et 4 forment un transect partant d'un peuplement adulte voisin (au Nord-Ouest) et traversant tout le verger à graine.

Nous avons calculé la proportion de pollen capté sur la ligne au niveau de chaque point.

- au cours de toute la campagne de piégeage ('T) ;

- au cours de la floraison locale $(7 / 5)$;

- au cours de cette dernière période sur les pièges exposés au Nord et à l'Fsst $(\mathrm{N}+\mathrm{E})$.

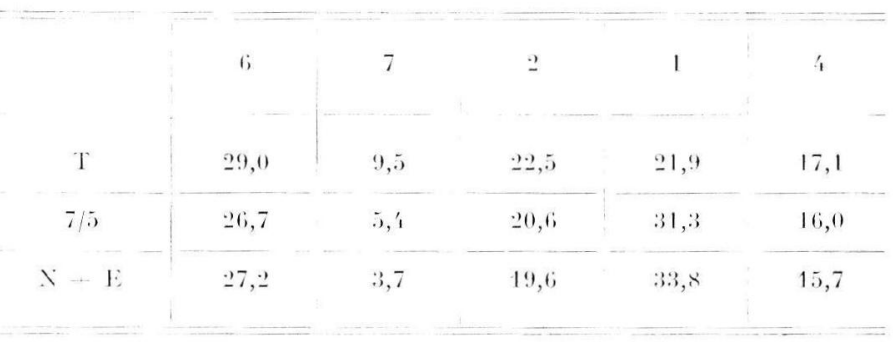

'Trois faits sont à remarquer :

- il y a sédimentation importante du pollen sur les lieux d'émission (point I). Cette quantité de pollen n'est pas impliquée dans le phénomène que nous étudions et n'est pas concerné par le modèle théorique précédemment défini. I1 en est de même pour les faibles quantités de pollen transportées à très longue distance.

- 1a lisière est pauvre en pollen (point 7);

- le maximum de pollen piégé se trouve aux alentours des points I et 2, la quantité au point 4 étant déjà la moitié de celle observée au centre du rerger.

4. 2.2. Etude des transects en 1971 sur les lignes de piégeage $A$ et $B$.

Nous ne rapporterons que les résultats au moment de la dissémination locale (1e $7 / 5)$.

Transect A en p. Ioo pour toutes les directions et seulement pour le pollen venant du Nord et de l'Fist $(\mathrm{N}+\mathrm{E})$.

\begin{tabular}{|c|c|c|c|c|c|c|c|c|}
\hline & $A 1$ & $A:$ & $\mathrm{A} 3$ & $A_{4}$ & $A ;$ & $A 6$ & $A 7$ & $A R$ \\
\hline $\mathrm{T}$ & $1: 3,9$ & 13,9 & 11,6 & 13,1 & 13,9 & 13,1 & 12,3 & 8,2 \\
\hline$x \quad 1:$ & 19,3 & 10,8 & $1: 3,2$ & 17,1 & 17,8 & 9,4 & 10,1 & 2,3 \\
\hline
\end{tabular}


De même pour le transect $B$

\begin{tabular}{|c|c|c|c|c|c|c|c|}
\hline & 13 1 & 132 & $1 ;: 3$ & $13^{\prime}$ & $B .7$ & 136 & B 7 \\
\hline $\mathrm{T}$ & $\because 2,1$ & 18,5 & $1.5,9$ & 11,9 & 7,7 & 12,5 & 11,4 \\
\hline$x+1:$ & 18,4 & $1: 3,: 3$ & $16, i^{\prime}$ & $1: 2,0$ & 9,5 & 15,4 & 15,0 \\
\hline
\end{tabular}

Les résultats sont illustrés par les courbes de la figure 2 .
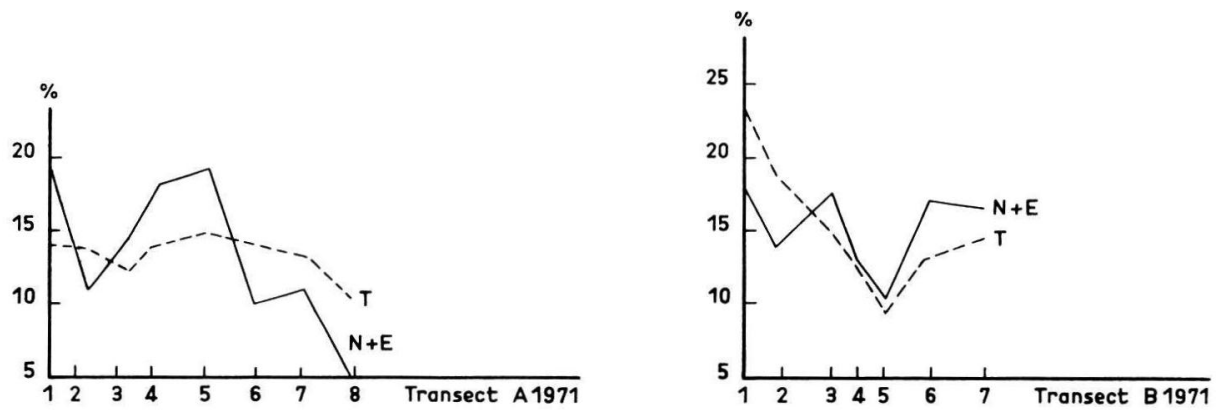

FIG. 2. - Dispersion du pollen sur le Verger a graines de Sore selon les transects A ef I3

Pollen dispersion in the seed orchard of Sore along the transects $A$ and $B$

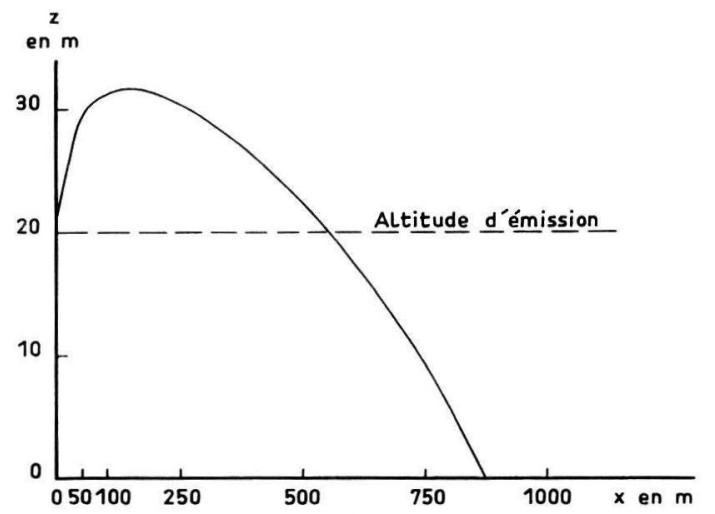

III. 2. - Représentation graphique selon l'équation de RomBAKis

Graphical representation according to RoMBakis équation

Pour ce qui est du transect $A$, nous retrouvons les résultats de I970. Le maximum de dépôt est constaté au niveau du point 5 , soit à 600 mètres environ du peuplement disséminateur. L'effet de lisière est retrouvé à I00-200 mètres environ du massif. Il existe toujours une sédimentation importante du pollen aux environs de la source. L,e nombre de grains piégés diminue très nettement à partir de 600 mètres. 
A I 200 mètres de l'émission, la quantité de pollen déposée n'est plus que le sixième de celle observée au niveau du maximum.

La courbe relative au transect B est plus complexe. Le peuplement pollinisateur forme un arc de cercle autour de la ligne B. Le maximum de dépôt se retrouve vers 600 mètres et la complexité de la courbe s'explique par la présence d'un petit massif situé tout à fait à 1'Est du verger et qui surcharge les points B 5, B 6 et peut-être B 7 .

\section{3. - Essai de cartographie de la pollution du verger (année 1972 et 1973}

A partir de 1972,25 points de piégeage régulièrement répartis sur la surface du verger (fig. I) ont été retenus. Nous espérons connaitre avec une meilleure approximation, la répartition du pollen " sauvage "s se déposant sur la surface du verger.

Les piégeages ont été répétés en I973, sur les mêmes emplacements. Un changement important est survenu cette année-là au niveau des peuplements avoisinant le verger. Au cours de l'hiver r972-I973 les peuplements adultes situés au Nord-Est du verger ont été abattus. L'observation des deux plans établis au cours des deux années successives (fig. 3 et 4 ) ont montré une zone de pollution intense de
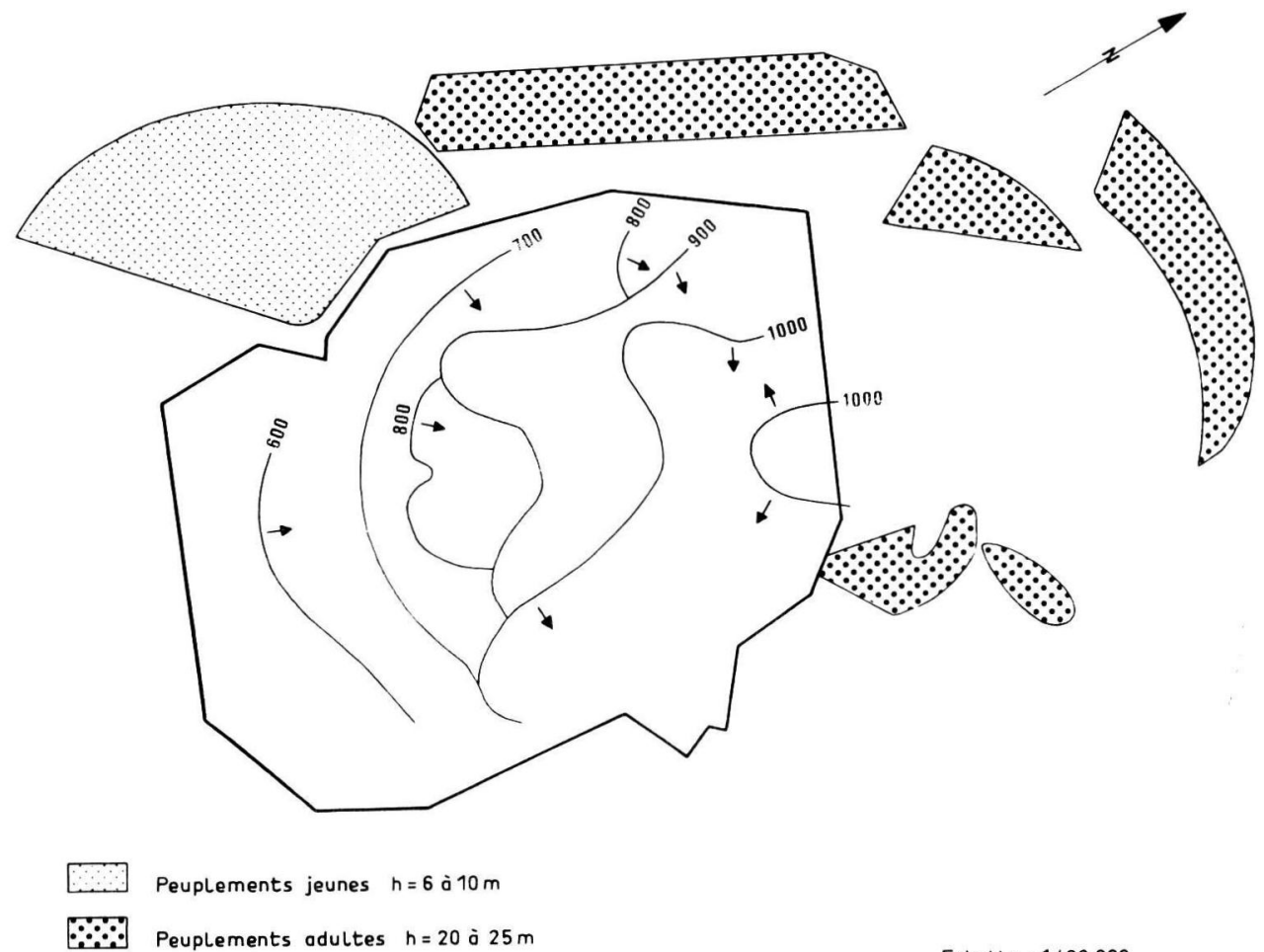

Peuplements jeunes $h=6$ à $10 \mathrm{~m}$

Peuplements adultes $h=20$ ò $25 \mathrm{~m}$

III. 3. - Plan de répartition de la masse pollinique sur le verger à graines de Sore (Landes) 1972. Toutes directions confondues 
toute la moitié Nord-Est du verger. Cette zone diminue sur toute sa portion Est, après l'abattage des peuplements voisins. La partie Sud du verger commence de plus à émettre du pollen se déposant essentiellement au Nord du verger.

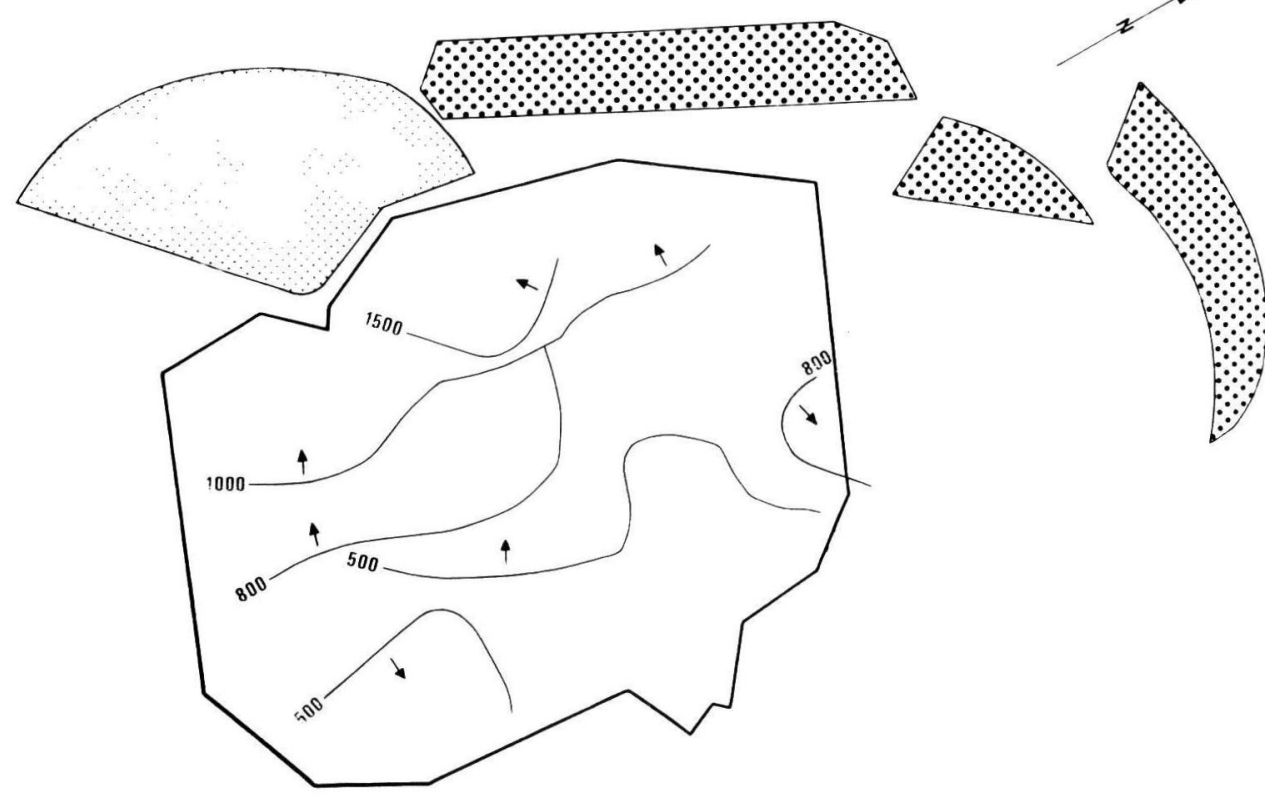

P... Peuplements jeunes $h=6$ ò $10 \mathrm{~m}$

$\because \because \quad$ Peuplements odultes $h=20$ ò $25 \mathrm{~m}$

Echelle : 1/20000

FIG. 4. - Plan de répartition de la masse pollinique sur le vergè à graines de Sore (Landes) 197.3. Toutes directions confondues

1ig. 4. - Repartition scheme of Pollen mass in the seed of Sore (Landes) 197.3

\section{5. - DISCUSSION}

5. I. - Dès I969, nous pouvions remarquer que la dissémination locale au sens strict, c'est-à-dire, intéressant les peuplements de pin maritime de la région de Sore, s'était étendue sur deux périodes de piégeage du 28 avril au 5 mai. Dans ce laps de temps où la réceptivité des fleurs femelles était maximale, $92 \mathrm{p}$. Ioo du pollen total est reçu, 3,2 p. Ioo étant piégé avant, 4,8 p. Ioo après. Le pollen impliqué dans un phénomène de dispersion lointaine ne représente donc qu'un peu plus du trentième de la quantité totale de pollen reçu au cours de la floraison régionale au sens strict. Ceci est vrai quand cette floraison est rapide, non perturbée par des conditions météorologiques défavorables, ce qui fut le cas en r970 et r97I. I e pollen reçu avant la floraison locale représente alors environ le cinquième de cette dernière. 
Le pollen impliqué dans des transports sur de longues distances est donc peu important. Son importance génétique devrait être nulle à cause des décalages régionaux de floraison dans les Landes (IL, L, I g63).

Si peu de preuves existent confirmant que du pollen d'origine lointaine n'est pas reçu en grande quantité pendant la période de dispersion locale, nous pouvons cependant admettre que cette fraction des retombées piégées est homogène sur toute la surface de la région et, au pire, est égale au minimum recueilli au cours de cette même période.

5. 2. - A l'époque de floraison des massifs environnant le verger à graine, la direction dominante du vent a évidemment changé d'une année à l'autre.

Cependant, quelle que soit l'annee, le pollen venant du Nord et de l'Est représente la plus grande partie :

\begin{tabular}{|c|c|c|c|c|c|}
\hline Annéc & 1969 & 1970 & 1971 & 1972 & 1973 \\
\hline $\begin{array}{l}\text { P. } 100 \text { pollen venant } \\
\text { de l'Est ........... }\end{array}$ & 66,000 & $\therefore 2,17$ & $6 y^{\prime}, 66$ & is, 59 & 47,70 \\
\hline
\end{tabular}

- Cette proportion diminue régulièrement alors que celle concernant les apports venant de l'Ouest augmente.

\begin{tabular}{c|cccc}
\hline 1969 & 1970 & 1971 & 1972 & $197 \%$ \\
\hline $7 \%$ & $6,89 \%$ & $13,69 \%$ & $32,719 \%$ & $27,69 \%$ \\
\hline
\end{tabular}

Or, la hauteur des peuplements situés directement à l'Ouest du verger n'était égale qu'à 9 mètres environ en 1969 .

La floraison mâle s'est intensifiée au fur et à mesure des pousses successives.

- De plus, les chiffres obtenus au niveau des pièges exposés au Sud sont nettement plus importants en 1973 .

\begin{tabular}{l|llll}
\hline 1969 & 1970 & 1971 & 1972 & 1973 \\
9,87 & 6,89 & 13,69 & 8,71 & 27,66 \\
\hline
\end{tabular}

En I973, la première tranche du verger à graine située sur la périphérie Sud-Esst et âgée de 6 ans, a commencé à fleurir.

- Si nous ajoutons que la coupe des peuplements adultes voisins situés à 
l'Est du verger à graine a profondément modifié l'allure générale de la contamina_ tion pollinique du verger, nous pouvons avancer que les peuplements voisins sont impliqués pour une large part dans la pollution générale du verger.

De I 969 à I972, ce sont les peuplements des secteurs Nord-Ouest, Nord, Nord-Esst qui ont constitué la source principale du pollen se déposant sur le verger.

Dans la formule (2) nous avons :

$$
\begin{aligned}
& \mathrm{A}=\frac{x \mathrm{C}^{2} \delta}{0,9 \mathrm{I} \mathrm{V}} \\
& x=600 \mathrm{mètres} \\
& \mathrm{C}=0, \mathrm{I} 7 \mathrm{~m} / \mathrm{s}
\end{aligned}
$$

Nous fixerons la vitesse du vent $\mathrm{V}$ à $2 \mathrm{~m} / \mathrm{s}$ ce qui est une valeur raisonnable pour la période considérée.

Nous calculons :

$$
\mathrm{A}=\frac{20,88}{\mathrm{I}, 82} \# \mathrm{II}, 5 \mathrm{~g} \cdot \mathrm{m} / \mathrm{cm} \mathrm{s}
$$

Or, le point d'émission (cime des arbres) étant situé à $\mathbf{I}_{5}-20 \mathrm{~m}$ environ, nous pourrions d'après les estimations des météorologistes fixer la valeur du coefficient d'échange à Io $g \cdot \mathrm{m} / \mathrm{cm} \mathrm{s}$. Les résultats sont donc parfaitement cohérents et le modèle de Rombakis semble s'adapter au problème d'une manière tout à fait satisfaisante, compte tenu de la précision des estimations, notamment de $x$.

En appliquant nos données numériques, la formule de base (I) devient :

$$
z=0,48 \sqrt{16,7} x-0,0083 x
$$

fonction dont nous pouvons établir la représentation graphique (fig. 2). Au début du phénomène, le centre du nuage s'élève, ce qui peut expliquer l'effet de lisière en plus des phénomènes de turbulence existant au niveau de la bordure du peuplement qui ont été étudiés par G. Gloyne (I954).

Les résultats expérimentaux, en particulier le long du transect $A$, nous donnent comme distance moyenne probable de dispersion 600 mètres environ.

La formule nous donnerait un peu plus (900 $\mathrm{m})$, mais cette valeur est directement proportionnelle au coefficient d'échange atmosphérique A que nous ne connaissons pas, avec exactitude et qui peut varier de plus de ro p. Ioo ainsi qu'à la vitesse du vent fortement variable.

5. 3. - Reprenons la répartition du pollen récolté sur les pièges exposés au Sud en 1973 .

Une cartographie rapide (fig. 5) nous montre que le maximum de chute du pollen émis par la région Sud-Est du verger se situe autour des points I, 3 et 2 de piégeage soit à 500 mètres environ du point d'émission. Des mesures exactes de vent ont été relevées grâce à un anémomètre à cupules.

La vitesse moyenne au cours des trois jours de dispersion intense a été de $2,32 \mathrm{~m} / \mathrm{s}$

$$
\mathrm{A}=8, \mathrm{I} 4 \mathrm{~g} \cdot \mathrm{m} / \mathrm{cm} \mathrm{s}
$$

inférieur a ce qui est trouvé précédemment, ce qui est normal puisque l'altitude d'émission est plus faible. 


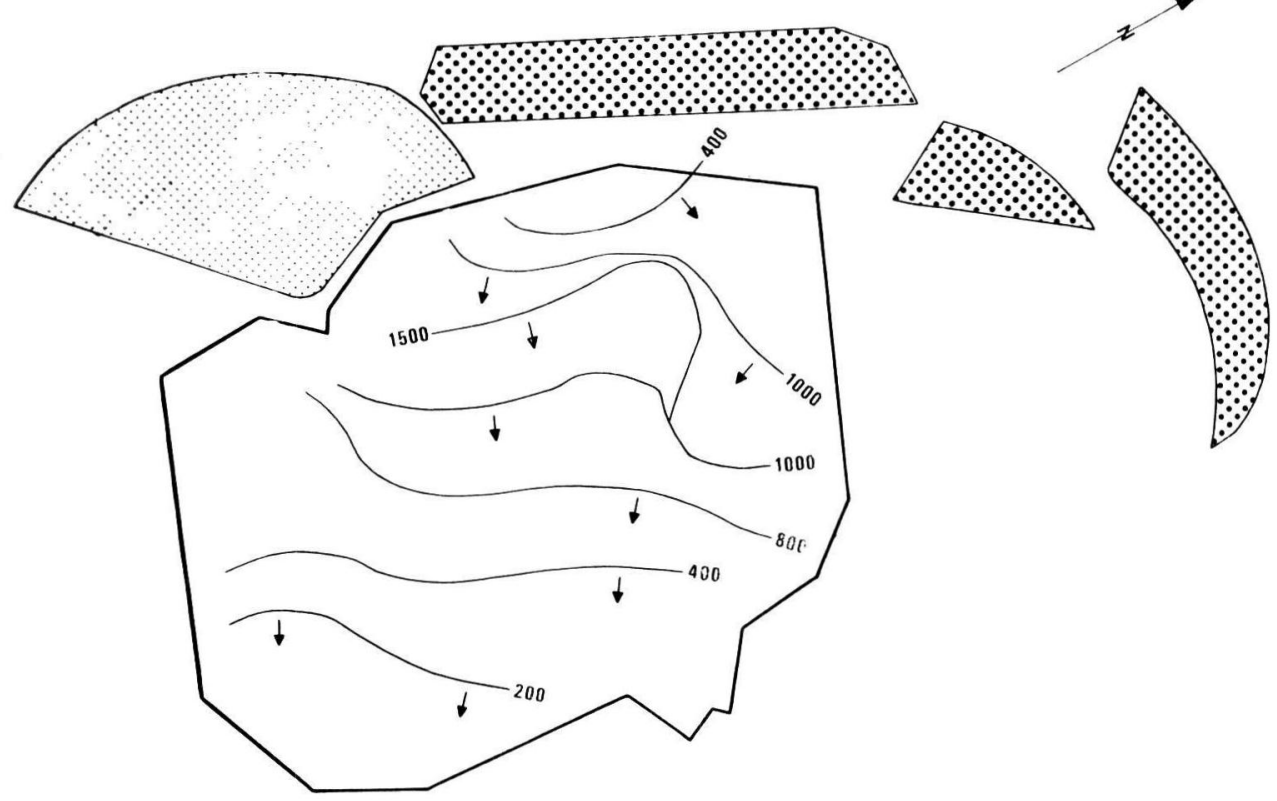

Peuplements jeunes $h=6$ ò $10 \mathrm{~m}$

$\because \because$ Peuplements adultes $h=20$ ò $25 \mathrm{~m}$

Echelle : $1 / 20000$

Fig. 5. - Répartition de la masse pollinique issue du sud-fst du verger à graines de Sore 1!r.:3

lic. 5. - Repartition of pollen mass coming from the South liast of seed orchard of sore 19:.3

\section{CONCLUSIONS}

Le modèle mathématique de Rombakis peut donc être utilisé pour étudier le phénomène de dispersion du pollen de pin maritime.

Nous pouvons estimer, selon la hauteur du peuplement disséminateur que la distance moyenne probable de dissémination est de 500 - I ooo $\mathrm{m}$.

I1 ne s'agit que d'un des paramètres caractérisant le phénomène global de dispersion. Seront intéressants à connaître, la répartition autour de cette distance moyenne probable et, par la suite, les proportions qui, dans un peuplement, existent entre le pollen endogène et exogène.

Lorsque nous considérons un point particulier du massif, ici le verger à graine de Sore, et plus particulièrement son centre, destiné à l'installation du matériel génétique ayant profité au maximum des efforts de sélection, l'apport extérieur est constitué grossièrement par :

- I/5 d'apport lointain, pouvant sans doute être éliminé à cause du décalage existant dans le massif au moment de la floraison et de la quantité du pollen venu des régions lointaines dont la viabilité est sans doute à peu près nulle. 
- 4/5 d'apport local dont la plupart est issu d'une région concentrique au verger de 500 à I ooo mètres de largeur.

Le terrain destiné à la constitution d'un verger à graine de pin maritime doit donc présenter une surface telle que ses dimensions minimum atteignent un kilomètre environ. Le verger à graine de Sore présente donc une surface juste suffisante.

Par contre, selon la force et la direction des vents, les tranches périphériques auront tendance à échanger du pollen entre elles, le centre du verger étant ainsi en partie protégé.

La contamination du verger à graine évoluera dans le temps. La distance moyenne probable de dissémination augmentant avec la taille de l'arbre (A et X augmentant au fur et à mesure que l'altitude d'émission augmente), le pollen émis par les arbres du verger restera en grande partie endogène au cours de ses premières années de production. Le poids génétique du pollen "sauvage " en sera donc diminué.

La portée de ce phénomène sera cependant réduite par le fait que les diverses tranches auront une croissance simultanée.

Les parties périphériques étant plus hautes, car plus âgées, elles serviront d'écran dans la mesure où les parties voisines extérieures au verger seront plus jeunes, de telle façon que sur une couronne de ioo mètres environ, la hauteur des cimes des arbres de la périphérie soit supérieure à la hauteur maximale probable atteinte par le nuage émis par les peuplements immédiatement voisins.

De toute façon, ces peuplements seront progressivement éliminés à mesure que le verger entrera en production.

Sur un plan général, lors de l'installation d'un verger à graine dans un massif, il est préférable que le voisinage ( $\mathrm{km}$ à la ronde environ) soit génétiquement connu et/ou de bonne qualité peuplé avec du matériel qui le soit. Il est peut-être possible d'allier l'amélioration d'une espèce par sélection individuelle et par choix d'une provenance intéressante en installant un verger à graine de semis dans une localité repérée pour ses peuplements constituant une telle provenance.

De plus, l'installation en zones concentriques et centripètes telle qu'elle est pratiquée à Sore, est parfaitement justifiée.

Il est peut-être possible d'installer un dispositif expérimental réunissant deux espèces, l'une servant de protection à l'autre. L'espèce propre à la région serait installée sous forme de verger à graine de semis. Ce verger à graine serait entouré par une plantation conservatoire de clones ou de provenances d'une autre espèce. Ce processus limiterait considérablement la contamination du verger à graines par du pollen extérieur mais en augmenterait considérablement les charge. de gestion et d'installation (pour un verger à graine tel que Sore, il serait nécessaire de planter un terrain d'une superficie totale de 530 hectares environ.

Enfin, le problème est compliqué par le fait que les décalages de floraison sont mal connus à l'intérieur du verger et que nous avons étudié le phénomène général du transport du nuage du polien après son émission. Or, nous savons déjà qu'il existe un phénomène de sédimentation locale sur le lieu d'émission, sans connaître son importance relative par rapport à l'ensemble de la production pollinique totale d'un peuplement tel que le verger à graines de Sore. 


\title{
REMERCIEMENTS
}

Les auteurs remercient Messieurs Arbez, Baradat, Gunaudeau et Mauge à qui ils doivent de précieux conseils pour l'établissement définitif de ce texte, ainsi que Mesdames Bertocchi, JIMENEZ et LAFORÊt qui ont effectué les nombreux comptages de grains de pollen nécessités par cette étude.

\section{SUMMARY}

\author{
EFFECTS OF THE DISPERSION OF POLLEN IN CONTAMINATING \\ A MARITIME PINE SEED ORCHARD (SORE, LANDES)

\section{AN EXPERIMENTAL STUDY}

The establishment of maritime pine seed orchards in the South West of France, requires more exact knowledge of the pollen dispersal.

The pollen dispersal measurements have been carried out in the seed orchard at Sore (Landes). Pollen measurements were made in I969-I973. The stations where the pollen traps were exposed, were distributed on the whole surface of the orchard or located along lines extending from the surrounding forest to the center of the open orchard area. A significant fact emerges : the surrounding stands give a low percentage of the pollen caught on the orchard site.

RомвакIs equation for the probable course of flight is a parabola. Now, a parabola is obtained with experimental data. The Romвakis model is a good representation of the natural course of the centre of gravity of the pollen cloud. The probable range of flight is directly proportional to the vertical mass exchange caused by turbulence and to the horizontal wind velocity, and inversly proportionnal to the square of velocity of fall in quiet air of the pollen grain. This probable range of flight is equal to $500-\mathrm{I}$ ooo $\mathrm{m}$ for the maritime pine. This is important for the seed orchard management.

\section{RÉFÉRENCES BIBLIOGRAPHIQUES}

BARADAT Ph., I970. A general method for computation of final genetic gains in the case of several generations of selection with a risk of pollution by foreign pollen. Meeting on "Sexual reproduction of Forest Trees ". Varparanta, Finland.

Baradat Ph., Illy G., Mauge J. P., Mendiboure P., r97o. Conception et réalisation d'un verger à graine test de descendances de pin maritime. Compte rendu d'activité de l'AFOCEL, I5-42.

Castaing J. Ph., Vergeron R., i973. Principes et méthodes d'étude expérimentale de la dispersion du pollen de pin maritime dans le massif landais Pollen et Spores, 15, 2, 255-280.

Durham O. C., 1946. The volumetric incidence of atmospheric allergens. III. Rate of fall of pollen grains in still air. J. Allergy, 17, 70-78.

Groyne R. W., i954. Some effects of shelterbelts upon local and microclimate. Forestry, 27.

Gregory P. Hi., I945. The dispersion of airbone spores. Trans. Brit. Mycol. Soc. 28, $26-72$.

Illy G., Sopexa J., ig63. La dispersion du pollen de pin maritime. Revue Forestiòre française, $\mathbf{1}$, 7-18.

Rombakis S., i947. Ueber die Verbreitung von Pflanzensamen und Sporen durch turbulente Luftströmungen. Z. Meteorol, 1, 359-363.

Sarvas R., 1962. Investigations on the flowering and seed crop of Pinus silvestris. Commun Inst. For. Fenn. 53, 4 , $19^{8}$ p.

Strand L., I957. Pollen dispersal. Silvae Genetica, 6 (5), I29-I68.

Sutton O. G., 1947. The problem of diffusion in the lower atmosphere. Quart. Jour. Roy. Met. Soc., 93, 257.

Wright J. W., I953. Pollen dispersion studies. Some pratical applications. J. For., 1, no 22. 